\section{Dark lumen MR-Colonography: initial experience}

\begin{abstract}
Purpose: The aim of this study was to assess a new strategy of MR colonography for the detection of colonic polyps/malignancy. The technique is based on a rectal enema with water in combination with the intravenous application of a paramagnetic contrast agent. Methods: Twelve subjects were included in this study either due to suspected colorectal tumors or for screening purposes. For MR colonography the colon was filled with approximately $3000 \mathrm{ml}$ of tap water while Gadobenate dimeglumine $(0.2 \mathrm{mmol} / \mathrm{kg})$ was injected intravenously. A T1w GRE sequence was acquired before and $75 \mathrm{sec}$ after the contrast administration only in prone position. Three patient underwent the "bright lumen" MRC as well. All MR examinations were performed at least five days prior to conventional colonoscopy and the results of both modalities were compared. Results: The colonic filling with water resulted in a homogeneously low signal throughout the colonic lumen in all twelve subjects. Intravenous injection of gadolinium caused avid enhancement of the colonic wall. Similarly lesions arising from the colonic wall enhanced avidly. Dark lumen MR colonography correctly identified five polyps in four patients. Bright lumen MRC showed in one patient false positive results. Conclusion: The new MR colonography concept based on a dark colonic lumen and a bright, contrast enhanced colonic wall turned out to be a promising alternative to the traditional MRC based on a bright lumen concept. The new technique suggests a higher accuracy in detecting polyps, a better evaluation of other parenchymal organs as well as a reduced time needed for data acquisition and analysis.
\end{abstract}

Key words: Magnetic resonance imaging (MRI) - Virtual endoscopy - Colon - Contrast medias

Dunkellumen-MR-Kolonographie: Erste Erfahrungen. Zielsetzung: Ziel dieser Studie war es, eine neue Technik der MRKolonographie zum Nachweis von Polypen/Malignomen zu evaluieren. Die Technik verwendet die rektale Applikation von Wasser zur Distension des Dickdarms in Kombination mit einer intravenösen Gabe eines gadoliniumhaltigen Kontrastmittels. Methoden: Zwölf Patienten wurden mittels MR-Kolonographie untersucht. Die Untersuchungen wur-den bei V.a. ein kolorektales Tumorleiden oder zum Screening durchgeführt. Zur Distension des Kolons wurde ein rektaler Einlauf mit $3000 \mathrm{ml}$ Wasser verabreicht, zusätzlich wurde Gadolinium Dimeglubine

Fortschr Röntgenstr 2001; 173: 785-789

(c) Georg Thieme Verlag Stuttgart · New York

ISSN 1438-9029
T. C. Lauenstein, C. U. Herborn, F. M. Vogt, S. C. Göhde, J. F. Debatin, S. G. Ruehm

Zentralinstitut für Röntgendiagnostik Universitätsklinikum Essen

$(0,2 \mathrm{mmol} / \mathrm{kg})$ intravenös injiziert. Die Akquisition einer $\mathrm{T}_{1}$-gewichteten 3D-GRE-Sequenz erfolgte vor und 75 Sekunden nach der intravenösen Kontrastmittelapplikation in Bauchlage des Patienten. Drei der zwölf Patienten wurden zusätzlich mittels der herkömmlichen MR-Kolonographie basierend auf einem hellen Darmlumen untersucht. Im Anschluss daran erhielten alle Patienten eine konventionelle Koloskopie. Ergebnisse: Bei allen zwölf untersuchten Patienten führte der rektale Einlauf mit Wasser zu einem homogen signalarmen Kolonlumen. Mit der intravenösen Applikation von Gadolinium konnte ein deutlicher Signalanstieg der Darmwand erreicht werden. Kolonpolypen zeigten ebenfalls eine deutliche Kontrastmittelaufnahme. Mit der neuen, auf einem dunklen Lumen basierenden MR-Kolonographie konnten fünf Polypen bei vier Patienten nachgewiesen werden. Diese Befunde wurden durch die Endoskopie bestätigt. Falschnegative Resultate kamen nicht vor. Die auf einem hellen Lumen basierende MR-Kolonographie zeigte jedoch bei einem Patienten ein falsch-positives Resultat. Schlussfolgerung: Die beschriebene Technik der MR-Kolonographie, welche auf einem dunklen Darmlumen und einer hellen Darmwand basiert, stellt eine vielversprechende Alternative zur herkömmlichen MR-Kolonographie mit hellem Darmlumen dar. Mit der neuen Technik können Kolonpolypen trotz einer Verkürzung von Untersuchungs- und Bildinterpretationszeit exakter detektiert werden. Zusätzlich können die übrigen abdominellen Organe genauer beurteilt werden.

Schlüsselwörter: Magnetresonanztomographie (MRT) - Virtuelle Endoskopie - Kolon - Kontrastmittel

\section{Introduction}

Colorectal cancer (CRC) remains the second leading cause of cancer mortality in western countries. Approximately $6 \%$ of the population will develop CRC during their lifetime [1]. The majority of colon cancers develop from non-malignant adenomas or polyps [2]. Thus, cancer screening programs targeting precancerous polyps with subsequent endoscopic polypectomy could potentially reduce the incidence and thus the mortality of colorectal cancer significantly.

Insufficient diagnostic accuracy and/or poor patient acceptance characterise most available colorectal screening modalities, including testing for occult fecal blood, conventional colonoscopy, or double-contrast barium enema [3 - 4]. Recently virtual colonography (VC), based on 3D CT or MR data sets has been propagated for colorectal screening. VC has been found to be 
highly sensitive for detecting colorectal polyps exceeding $8 \mathrm{~mm}$ in size $[5,6]$. Despite high diagnostic accuracy, the considerable exposure to ionising radiation casts a shadow over the future of CT colonography as a screening exam for colorectal cancer [7]. Hence, efforts have been focussed on MR colonography (MRC).

To date, MRC has been based upon the administration of a rectal enema containing paramagnetic contrast. On 3D gradient echo data sets only the contrast-containing colonic lumen is bright whereas the surrounding tissues including colonic wall and polyps remain low in signal intensity. Hence the technique has been referred to as 'bright lumen' MRC. Polypoid colonic masses appear as dark filling defects within the bright colonic lumen an appearance which is difficult to differentiate from residual fecal material and/or small pockets of air. To avoid false positive findings induced by residual stool, patients are required to rigorously cleanse their colon prior to the exam. To compensate for the presence of residual air, the 3D acquisition is performed in both the prone and supine positions.

In this communication we describe our initial experience with a simplified, less costly variation on MR-colonography - MRI of the contrast-enhanced colonic wall. The technique is based on the acquisition of a 3D gradient echo sequence collected after administration of a rectal water-enema and an intravenous injection of paramagnetic contrast. The colonic wall as well as masses arising from it brightly enhance and are thus easily delineated against the background of dark surrounding tissues and a dark colonic lumen - hence ‘dark lumen’ MRC.

\section{Materials and methods}

\section{'Dark lumen' MRC: technique}

Following standard preparation for bowel cleansing (oral ingestion of 4 L Golytely, Braintree Laboratories, Braintree, Massachusetts) MR examinations were performed on a $1.5 \mathrm{~T}$ MR system (Magnetom Sonata, Siemens Medical Systems, Erlangen, Germany). A combination of two surface coils were used in conjunction with the built-in spine array coil for signal reception to permit coverage of the entire colon. To minimize bowel peristalsis, $40 \mathrm{mg}$ of scopolamine (Buscopan; Boehringer Ingelheim, Germany) were injected intravenously. Following placement of a rectal enema tube (E-Z-Em, Westbury, NY), the colon was filled with $3000 \mathrm{ml}$ of warm tap water. To ensure safe and complete filling, the administration of the enema was monitored using a fast 2D TrueFISP sequence (TR/TE/flip 3.2/ $1.6 / 70^{\circ}$; slice thickness $5 \mathrm{~mm}$ ) which allowed for the acquisition of one image every three seconds. Once complete filling and distension of the colon was assured, a first 'pre-contrast' T1w 3D gradient echo data set was collected. Data acquisition was performed with the patient in the prone position, only. For the $3 \mathrm{D}$ sequence the following parameters were used: TR/TE 1.64/ $0.6 \mathrm{~ms}$, flip angle $15^{\circ}$, field of view (FOV) 450 x $450 \mathrm{~mm}$, matrix $512 \times 460$, effective slice thickness $1.57 \mathrm{~mm}$. Subsequently paramagnetic contrast (gadobenate dimeglumine, Gd-BOPTA, Multihance, Bracco, Italy) was administered i.v. at a dosage of $0.2 \mathrm{mmol} / \mathrm{kg}$ and a flow rate of $3.5 \mathrm{ml} / \mathrm{s}$. After a delay of $75 \mathrm{~s}$, the 'pre-contrast' 3D acquisition was repeated with identical imaging parameters. The 3D data set was collected breahthheld in $23 \mathrm{~s}$.

\section{Patients}

MRI of the colonic wall was performed on 12 subjects ( 8 men, 4 women, age range 44 - 76 years, mean age 60.2 years) in whom a colorectal mass was suspected due to positive family history $(n=3)$ or a positive fecal occult blood test $(n=9)$. The study was performed in accordance with all guidelines set forth by the local ethical committee and all patients signed informed consent.

In addition to MRI of the colonic wall all patients underwent conventional colonoscopy performed within five to fourteen days following the MR exam. Besides, three subjects agreed to undergo MR-colonography based on the published 'bright lumen' protocol [8]. These exams were performed seven days following the 'dark lumen' MRC, on the same MR system, using identical patient and coil positioning, as well as the same 3D gradient echo sequence for display of the colon. In contrast to the 'dark lumen' MRC protocol, Gd-DTPA was added to the rectal water enema $(1: 100)$ and no intravenous contrast agent was applied.

\section{Data Analysis}

All MRI exams were evaluated by two experienced radiologists. Analysis was based on individual source images, multiplanar reformations and virtual endoscopic renderings. Signal intensities were measured in Regions-of-interest (ROI) positioned within the walls of the ascending, transverse, descending and sigmoid colon as well as within all mass lesions on both unenhanced and enhanced 3D GRE images. Signal-to-Noise Ratios (SNR) were calculated in the usual manner using the following formula: SNR = SI (colonic wall)/noise defined as the standard deviation of an ROI measurement outside the subject. All MR findings were compared to those obtained with conventional endoscopy.

\section{Results}

'Dark lumen' MRC, including placement of the rectal tube and colonic filling with warm tap water was well-tolerated by all twelve subjects. All twelve exams were considered diagnostic. The in-room time ranged between 10 and 15 minutes (mean 12 minutes). Image analysis time amounted to $10 \pm 4$ minutes.

Five polyps ranging in diameter between 7 and $12 \mathrm{~mm}$ were detected with 'dark lumen' MRC (Fig. 1). All five lesions were confirmed by conventional colonoscopy and subsequent polypectomy was performed. There were no false negative findings.

On 'bright lumen' MRC, performed in addition in only three patients, three polyps were seen in two patients. One lesion corresponded to a polyp seen on 'dark lumen' MRC as well as at colonoscopy. Two lesions identified in one patient did not have a correlate on either 'dark lumen' MRC or conventional colonoscopy. The two false positive findings were retrospectively interpreted as either residual air bubbles or residual stool adherent to the colonic wall .

The intravenous administration of paramagnetic contrast resulted in an average SNR increase within the colonic wall of $170 \%$ from 9.2 to $24.8 \pm 2.6$. This difference was statistically 

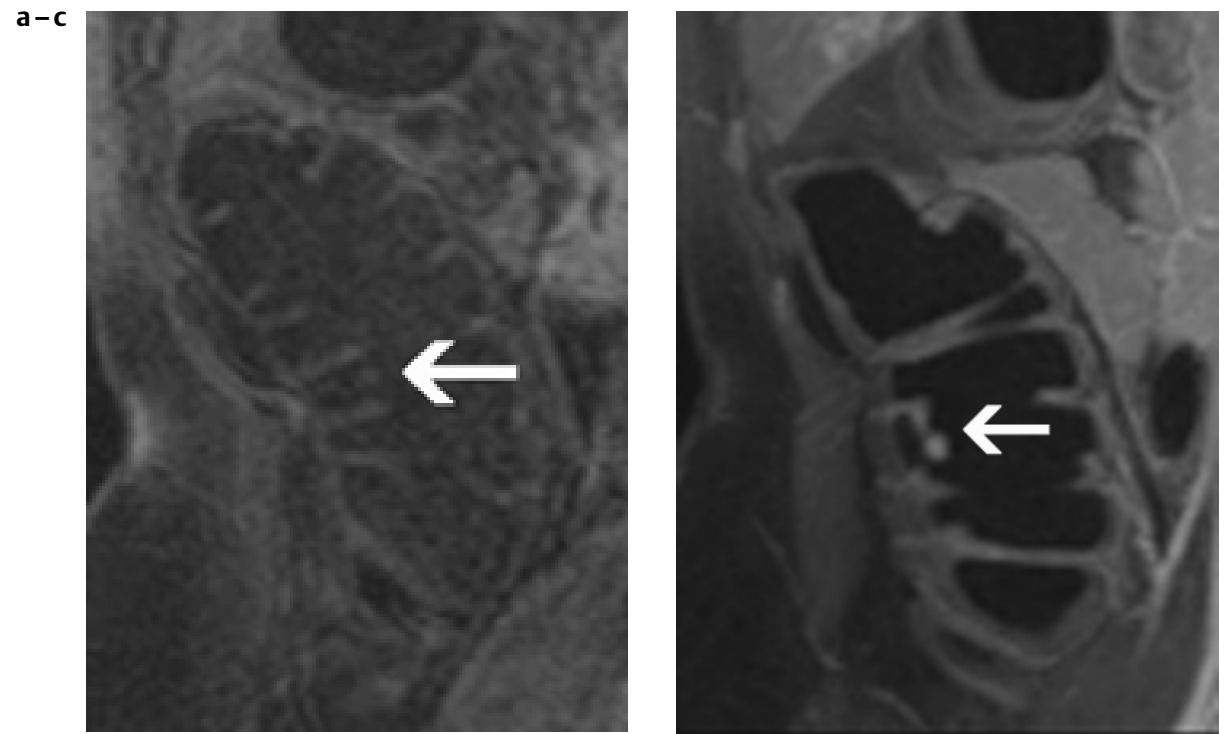

Fig. 1 49-year-old woman referred for MRC and conventional colonoscopy due to positive occult fecal blood test. $10 \mathrm{~mm}$ polyp could be detected in the ascending colon based on the contrast uptake in (b) (arrow) compared to the corresponding native sequence show in (a) (arrow). Diagnosis was confirmed by virtual endoscopic rendering (c) as well as by conventional colonoscopy. $\mathbf{a}, \mathbf{b}$

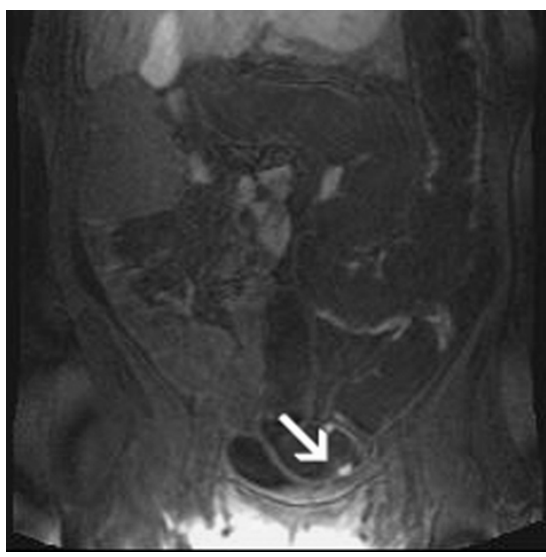

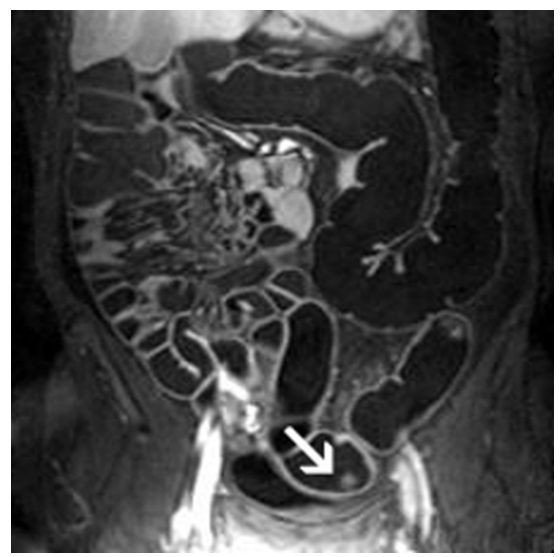

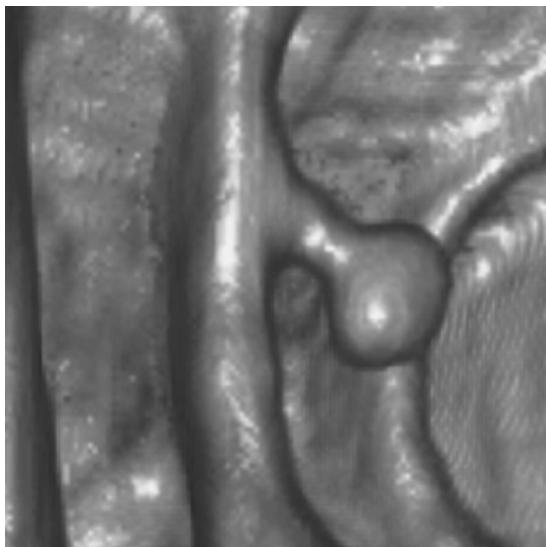

Fig. 2 61-year-old female patient undergoing MRC for colorectal cancer screening. Polyp-simulating protrusion in the sigmoid colon (b, arrow) turned out to be residual stool because of the same signal intensity compared to native scan (a, arrow). Subsequent conventional colonoscopy confirmed absence of colorectal pathologies. significant $(\mathrm{p}<0.001)$. Polyps revealed even more enhancement with signal intensities increasing by $306 \%$ from $8.9 \pm 1.6$ to 36.1 \pm 3.9. Lack of contrast enhancement correctly identified three bright "lesions" as residual stool (Fig. 2).

In addition, 'dark lumen' contrast-enhanced MRC revealed four extra-intestinal lesions: two renal cysts in two patients, a single hepatic hemangioma in one patient, and an aortic abdominal aneurysm measuring $4 \mathrm{~cm}$ in diameter in another patient.

\section{Discussion}

The preliminary experience documented in this communication suggests that 'dark lumen' MRC works well. The technique is well tolerated and appears highly accurate regarding the detection of colorectal masses - all 5 polyps were readily identified. Compared to 'bright lumen' MRC which has been extensively evaluated in the past, 'dark lumen' MRC harbors considerable advantages including reduced cost, reduced examination and post-processing times, as well as potentially higher diagnostic accuracy and confidence.
'Bright lumen' MRC, which has been shown to be accurate in detecting colorectal polyps larger than $8 \mathrm{~mm}$ in size, requires the administration of a gadolinium-containing rectal enema $[6,8,9]$. Although most authors suggest a mixture of $1: 100$ [6], some studies recommend the use of a $1: 50 \mathrm{Gd} /$ water dilution [8]. Assuming a colonic volume of $3000 \mathrm{ml}$, between 30 and $60 \mathrm{ml}$ of costly paramagnetic contrast are needed for the rectal enema alone. In addition, most 'bright lumen' MRC protocols call for the additional administration of paramagnetic contrast in a dose of $0.1 \mathrm{mmol} / \mathrm{kg}$ for better assessment of surrounding organs such as the liver. The 'dark lumen' MR-colonography approach on the other hand requires merely a single intravenous injection of less than $30 \mathrm{ml}$ of paramagnetic contrast for a subject weighing $70 \mathrm{~kg}$. No additional injection of contrast is required for concomitant assessment of parenchymal organs.

To compensate for residual air pockets, which obscure the outline of the colonic wall, 'bright lumen' MRC requires the collection of two data sets: one obtained in the prone and a second obtained in the supine patient position. Turning the patient during the exam is cumbersome and can be associated 
with considerable time delays. Occasionally the patient moves so much that a new landmark is required. In any case, a new localizing sequence is required to assure full coverage of the colon in the subsequent 3D acquisition. During this delay, contrast frequently escapes from the colon into the small bowel. As a result the colon looses distension and the resultant data set is of reduced diagnostic quality. 'Dark blood' MRC obviates the need for a 3D acquisition in a second patient position. Since air is signalless on all sequences, its appearance on heavily T1-weighted 3D GRE images is identical to water, which is used to distend the colon. The enhancing colonic wall and mass lesions arising from it are easily differentiated. Thus the time for both the actual exam as well as image interpretation is considerably reduced amounting to less than 30 minutes.

The detection of colorectal lesions with 'bright lumen' MRC relies on the visualization of filling defects. Differential considerations for such a filling defect include air bubbles as well as residual fecal material. Collecting two data sets in the prone and supine patient position allows the use of 'motion' as a differentiating criterion. Only those lesions that remain in the same position are considered a true polyp. Unfortunately this differentiating criteria can introduce severe errors, both regarding false negatives and false positives. Thus polyps with a long stalk may move sufficiently to impress as a moving air bubble or more likely residual stool, while stool adherent to the colonic wall may not move at all and thus falsely impress as a polyp. This was the case in one patient examined in the current collective - based on the 'bright lumen' technique two small polyps were identified, which had no correlate on either 'dark lumen' MRC or conventional colonoscopy.

All techniques for virtual colonography, regardless whether based on CT or MRI are handicapped by residual stool. The proposed 'dark lumen' technique copes with this problem in a simple manner: if the lesion enhances it is a polyp, if it does not enhance it represents stool. Suspicious appearing lesions are analyzed by comparing signal intensities on the pre- and post-contrast images. Lesions identified in this limited number of patients enhanced in average by more than $300 \%$. Comparing post- to pre-contrast data sets is crucial, as stool can be quite bright on T1-weighted images. The presence of iron and manganese is implicated as the cause for the bright signal within stool. If analysis were limited to the post-contrast data set, bright stool could be misinterpreted as a polyp. Comparison with the pre-contrast images documents the lack of contrast enhancement which assures the correct diagnosis. In the current study, several patients exhibited bright stool which was readily identified as such based on assessment of the precontrast images (Fig. 2).

Enhancement of colorectal masses following the intravenous administration of contrast has been documented before in conjunction with MR-colonography [10] and CT colonography [11]. The use of intravenously administered contrast material had significantly improved reader confidence in the assessment of bowel wall conspicuity and the ability of CT colonography to depict medium polyps in suboptimally prepared colons. Interestingly, the enhancement observed within polyps exceeded the increase determined within the colonic wall. In view of the very limited number of lesions, the reliability of this observation remains unclear. If proven true, this difference may aid in differentiating even very small polyps from thickened haustral folds.

A further advantage of 'dark lumen' MRC relates to the fact that it permits direct analysis of the bowel wall. This might facilitate the evaluation of inflammatory changes in patients with Crohńs disease. Increased contrast uptake and bowel wall thickening, as documented on contrast-enhanced T1-weighted images has already been shown to correlate well with the degree of inflammation in the small bowel [12]. Hence, the 'dark lumen' approach may indeed amplify the list of indications for MRC in the future to also encompass inflammatory bowel disease.

Finally, the intravenous application of paramagnetic contrast permits a more comprehensive assessment of parenchymal abdominal organs contained within the field of view. By combining pre- and post-contrast T1-weighted imaging, the liver can be accurately evaluated regarding the presence and type of concomitant disease. Accordingly, a hepatic hemangioma was not merely detected but immediately characterized as such on the contrast-enhanced scan. Not only hepatic lesions, but also vascular structures can be interpreted with more confidence. Thus one patient with an abdominal aortic aneurysm was readily identified.

'Dark blood' MRC also offers new perspectives regarding optimization of bowel distention. Although the administration of water as a rectal enema does not adversely effect patient comfort in most cases, a modified strategy could be based on the application of gas like $\mathrm{CO}_{2}[13]$. The gas is signalless and would thus easily permit delineation of the contrast-enhanced colonic wall and masses. Eventually, the technique may also offer new perspectives for 'fecal tagging' [14]. If the signal of stool could be reliably nulled, cleansing of the colon prior to the exam would no longer be necessary. Preliminary experiments using various orally applied contrast agents appear promising [15].

\section{Conclusion}

'Dark lumen' MR colonography is based on the contrast enhancement of the colonic wall and masses arising from it. Compared to 'bright lumen' MRC, the technique appears to enhance diagnostic accuracy and confidence, while at the same time reducing cost and shortening exam as well as postprocessing times.

\section{Literatur}

${ }^{1}$ Neuhaus H. Screening for colorectal cancer in Germany: guidelines and reality. Endoscopy 1999; 31: 468-470

2 O'Brien MJ, Winawer SJ, Zauber AG, Gottlieb LS, Sternberg SS, Diaz B, Dickersin GR, Ewing S, Geller S, Kasimian D. The National Polyp Study. Patient and polyp characteristics associated with highgrade dysplasia in colorectal adenomas. Gastroenterology 1990; 98: $371-379$

${ }^{3}$ Frommer DJ. What's new in colorectal cancer screening? J Gastroenterol and Hepatol 1998; 13: 528-533

${ }^{4}$ Ahlquist DA, Wieland HS, Moertel CG, et al. Accuracy of fecal occult blood screening for colorectal neoplasia. A prospective study using Hemoccult and HemoQuant tests. JAMA 1993; 269: $1262-$ 1267 
${ }^{5}$ Fenlon HM, Nunes DP, Schroy PC 3rd, Barish MA, Clarke PD, Ferrucci JT. A comparison of virtual and conventional colonoscopy for the detection of colorectal polyps. N Engl J Med 1999; 341: $1496-1503$

${ }^{6}$ Pappalardo G, Polettini E, Frattaroli FM, et al. Magnetic resonance colonography versus conventional colonoscopy for the detection of colonic endoluminal lesions. Gastroenterology 2000; 119: $300-304$

${ }^{7}$ Becker CR, Schatzl M, Feist H, et al. Radiation exposure during CT examination of thorax and abdomen. Comparison of sequential, spiral and electron beam computed tomography. Radiologe 1998; 38: $726-729$

${ }^{8}$ Luboldt W, Bauerfeind P, Wildermuth S, Marincek B, Fried M, Debatin JF. Colonic masses: detection with MR colonography. Radiology 2000; 216: 383-388

${ }^{9}$ Saar B, Heverhagen JT, Obst T, Berthold LD, Kopp I, Klose KJ, Wagner HJ. Magnetic resonance colonography and virtual magnetic resonance colonoscopy with the 1.0-T system: a feasibility study. Invest Radiol 2000; 35: $521-526$

${ }^{10}$ Luboldt W, Steiner P, Bauerfeind P, Pelkonen P, Debatin JF. Detection of mass lesions with MR colonography. Radiology 1998; 207: 59-65

${ }^{11}$ Morrin MM, Farrell RJ, Kruskal JB, Reynolds K, McGee JB, Raptopoulos V. Utility of intravenously administered contrast material at CT colonography. Radiology 2000; 217: 765 - 771

12 Marcos HB, Semelka RC. Evaluation of Crohn's disease using halffourier RARE and gadolinium-enhanced SGE sequences: initial results. Magn Reson Imaging 2000; 18: 263-268

${ }^{13}$ Lomas DJ, Sood RR, Graves MJ, Miller R, Hall NR, Dixon AK. Colon carcinoma: MR imaging with $\mathrm{CO}_{2}$ enema. Radiology 2001; 219: $558-562$

${ }^{14}$ Weishaupt D, Patak MA, Fröhlich J, Rühm SG, Debatin JF. Faecal tagging to avoid colonic cleaning before MRI colonography. Lancet 1999; 354: $835-836$

${ }^{15}$ Lauenstein T, Schoenfelder D, Bosk S, Debatin JF. Fecal tagging als Methode zur Verbesserung der Patientenakzeptanz. Deutscher Röntgenkongress 2001

\section{Dr. med. Thomas C. Lauenstein}

Zentralinstitut für Röntgendiagnostik Universitätsklinikum Essen

Hufelandstraße 55

45122 Essen

Tel. ++49 2017231576

Fax ++49 2017231526

E-mail: thomas.lauenstein@uni-essen.de 